\title{
Polyclonal Pulmonary Tuberculosis Infections and Risk for Multidrug Resistance, Lima, Peru
}

\section{Ruvandhi R. Nathavitharana, ${ }^{1}$ Cynthia X. Shi, ${ }^{1}$ Leonid Chindelevitch, Roger Calderon, Zibiao Zhang, Jerome T. Galea, Carmen Contreras, Rosa Yataco, Leonid Lecca, Mercedes C. Becerra, Megan B. Murray, Ted Cohen}

Because within-host Mycobacterium tuberculosis diversity complicates diagnosis and treatment of tuberculosis (TB), we measured diversity prevalence and associated factors among 3,098 pulmonary TB patients in Lima, Peru. The 161 patients with polyclonal infection were more likely than the 115 with clonal or the 2,822 with simple infections to have multidrug-resistant TB.

$\mathrm{W}$ ithin-host heterogeneity of Mycobacterium tuberculosis infection is increasingly recognized as an obstacle for the accurate diagnosis (1) and effective treatment (2) of tuberculosis (TB) and may complicate the control of TB in communities (3). Within-host heterogeneity may arise through 2 mechanisms: 1) by reinfection or simultaneous infection with multiple strains, which results in a polyclonal (mixed) infection, or 2) by accumulation of mutations, which results in clonal heterogeneity (4). The treatment challenge posed by within-host heterogeneity has been most clearly demonstrated for infections with drug-susceptible and drug-resistant variants (5). The relatively high prevalence of multidrug-resistant (MDR) TB in Peru $(\approx 6 \%$ among new case-patients and $21 \%$ among retreatment case-patients) (6) places increased stress on the healthcare system.

Our main objectives were to estimate the prevalence of within-host $M$. tuberculosis heterogeneity at the time of treatment initiation in a large cohort of pulmonary TB patients in Peru and to determine if factors measurable at the baseline visit were associated with complex infections (7). To determine whether our insights were sensitive to

Author affiliations: Beth Israel Deaconess Medical Center, Boston, Massachusetts, USA, and Imperial College London, London, UK (R.R. Nathavitharana); Yale School of Public Health, New Haven, Connecticut, USA (C.X. Shi, T. Cohen); Simon Fraser University, Burnaby, British Columbia, Canada (L. Chindelevitch); Socios En Salud Sucursal Peru, Lima, Peru (R. Calderon, J.T. Galea, C. Contreras, R. Yataco, L. Lecca); Harvard Medical School, Boston (Z. Zhang, J.T. Galea, L. Lecca, M.C. Becerra, M.B. Murray)

DOI: https://doi.org/10.3201/eid2311.170077 the method used for distinguishing between classes of heterogeneous infections, we used a newly described method (classifier of tandem repeats [ClassTR]) (8), which uses 24-loci mycobacterial interspersed repetitive units-variable number of tandem repeats (MIRU-VNTR) data to distinguish polyclonal and clonal infections, and we compared these findings with an analysis based on the standard threshold-based approach (9).

\section{The Study}

During September 2009-August 2012, we attempted to enroll all adults ( $>15$ years of age) with a diagnosis of incident pulmonary TB from 106 healthcare centers in Lima, Peru; details of the study design have been reported previously (7). We recorded baseline data on demographics, medical history, and results of drug susceptibility testing (DST) for rifampin, isoniazid, streptomycin, ethambutol, and pyrazinamide. We restricted our analysis to pretreatment samples and data from participants with culture-positive TB from whom sufficient mycobacterial DNA could be successfully obtained from the baseline sample to perform MIRU-VNTR typing.

All enrolled index case-patients and household contacts evaluated for active TB were assessed by sputum smear microscopy with Ziehl-Neelsen staining and culture on solid Lowenstein-Jensen medium. Initial DST was performed by using the proportion method on LowensteinJensen medium; second-line DST was performed by using the proportion method on Middlebrook $7 \mathrm{H} 11$ agar. We shipped $100 \mu \mathrm{L}$ of the lysate from suspensions of mycobacterial colonies harvested from Lowenstein-Jensen slants to Genoscreen (Institute Pasteur, Lille, France) for 24-loci MIRU-VNTR typing.

The standard threshold approach for classifying complex infections by using MIRU-VNTR data classifies patterns with $>1$ band (i.e., repeat copy number) at a single locus as clonal infections and patterns with $>1$ band at multiple loci as polyclonal infections (9). To better distinguish between clonal and polyclonal infections, we used an alternative method called ClassTR, which leverages additional information about differences in loci copy numbers and from other strains present in the population (8). In simulation studies, ClassTR more accurately distinguished between these 2 mechanisms of within-host diversity than did the threshold approach (8).

${ }^{1}$ These authors contributed equally to this article. 
To understand whether our findings were robust to the classification approach used, we adopted ClassTR for our main analysis, but we also repeated all analyses with the threshold approach. We used univariable and multivariable multinomial logistic regression to identify baseline factors independently associated with having a clonal or a polyclonal infection, setting simple infection as the referent. Analysis was limited to complete cases. Co-linearity was assessed by calculating variance inflation factors, and $\mathrm{p}<0.05$ was considered statistically significant. Co-linear variables were removed to produce the final multivariable model. Statistical analyses were conducted in R version 3.3 (http://www.R-project. org). Research ethics committees in Peru and Boston approved the study.

We analyzed results for 3,098 participants. Most participants were $<35$ years of age $(64.8 \%)$ and male $(61.9 \%)$; $108(3.5 \%)$ were known to be HIV infected. Nearly a fifth $(18.8 \%)$ of participants reported a prior history of $\mathrm{TB}$, and $78(2.5 \%)$ reported having received a course of isoniazid chemoprophylaxis. A total of 375 (12.1\%) participants had MDR-TB, 288 (9.3\%) had isoniazid or rifampin monoresistance, and $357(11.5 \%)$ had other resistance patterns (predominantly streptomycin resistance). A total of 2,822 (91.1\%) participants had simple infections (i.e., no evidence of within-host heterogeneity by MIRUVNTR), and the remaining 276 (8.9\%) had evidence of within-host heterogeneity. Using ClassTR, we classified $161(5.2 \%)$ infections as polyclonal and $115(3.7 \%)$ as clonal (Table 1).

Multivariable multinomial logistic regression results associated polyclonal infection with multidrug resistance (adjusted odds ratio $1.66,95 \%$ CI $1.05-2.62 ; \mathrm{p}=$ 0.03 ) and other drug resistance (adjusted odds ratio 1.97, $95 \%$ CI $1.27-3.06 ; p=0.002$ ) (Table 2). No factors were significantly associated with clonal infection in either
Table 1. Mycobacterium tuberculosis resistance patterns among patients with pulmonary TB, Lima, Peru, September 2009August 2012*

\begin{tabular}{|c|c|c|c|}
\hline Resistance $†$ & $\begin{array}{c}\text { Simple, no. } \\
(\%)\end{array}$ & $\begin{array}{c}\text { Clonal, no. } \\
(\%)\end{array}$ & $\begin{array}{c}\text { Polyclonal, no. } \\
(\%)\end{array}$ \\
\hline Pansensitive & 1,917 (67.9) & $73(63.5)$ & $88(54.7)$ \\
\hline $\begin{array}{l}\text { INH or RIF } \\
\text { resistance }\end{array}$ & $260(9.2)$ & $11(9.6)$ & $17(10.6)$ \\
\hline Multidrug & $333(11.8)$ & $15(13.0)$ & $27(16.8)$ \\
\hline Other & 312 (11.1) & 16 (13.9) & $29(18.0)$ \\
\hline Total & 2,822 & 115 & 161 \\
\hline \multicolumn{4}{|c|}{$\begin{array}{l}{ }^{*} \text { Mycobacterium tuberculosis strain type determined by classifier of } \\
\text { tandem repeats. INH, isoniazid; RIF, rifampin; TB, tuberculosis. } \\
\text { †Drug susceptibility testing was performed for RIF, INH, streptomycin, } \\
\text { ethambutol, and pyrazinamide. }\end{array}$} \\
\hline
\end{tabular}

univariable or multivariable analysis. These associations were largely preserved when we repeated the analysis by using the threshold classification approach (online Technical Appendix Tables 1, 2, https://wwwnc.cdc.gov/EID/ article/23/11/17-0077-Techapp1.pdf).

\section{Conclusions}

Among a large cohort of pulmonary TB patients in Lima, Peru, we found evidence of within-host $M$. tuberculosis diversity at the time of treatment initiation in $\approx 9 \%$. The ClassTR approach for classification based on MIRU-VNTR typing indicated that $5.2 \%$ of patients had polyclonal infections and $3.7 \%$ had clonal infections.

Polyclonal infections were positively associated with multidrug resistance and other drug-resistance patterns. When we used a 2-sided exact binomial test to test the hypothesis that the risk for multidrug resistance among participants with polyclonal infection (using the observed fraction 27/161) differed from that expected with infection by 2 randomly selected strains, calculated as $1-(1-348 / 2,937)^{2}$, we obtained a $p$ value of 0.11 . This $p$ value suggests that, at least in this setting, the association between polyclonal infection and multidrug resistance cannot be attributed to

\begin{tabular}{|c|c|c|c|c|}
\hline Characteristic & Clonal aOR $(95 \% \mathrm{Cl}), \mathrm{n}=115$ & $p$ value & Polyclonal aOR $(95 \% \mathrm{Cl}), \mathrm{n}=161$ & $p$ value \\
\hline \multicolumn{5}{|l|}{ Age, y } \\
\hline $15-24$ & Referent & & Referent & \\
\hline $25-34$ & $1.21(0.75-1.96)$ & 0.44 & $1.40(0.93-2.11)$ & 0.11 \\
\hline $35-44$ & $1.16(0.64-2.11)$ & 0.62 & $1.42(0.87-2.31)$ & 0.16 \\
\hline$\geq 45$ & $1.33(0.79-2.23)$ & 0.29 & $1.22(0.77-1.95)$ & 0.40 \\
\hline Male sex & $0.93(0.63-1.38)$ & 0.72 & $1.10(0.78-1.55)$ & 0.59 \\
\hline Previous TB & $0.82(0.48-1.37)$ & 0.45 & $1.27(0.86-1.86)$ & 0.23 \\
\hline Previous INH receipt & $1.51(0.54-4.27)$ & 0.44 & $1.84(0.82-4.15)$ & 0.14 \\
\hline HIV infection & $0.99(0.35-2.78)$ & 0.98 & $1.12(0.50-2.49)$ & 0.79 \\
\hline$\geq 1$ chronic disease & $0.90(0.55-1.46)$ & 0.66 & $0.97(0.64-1.46)$ & 0.88 \\
\hline Hospitalized & $1.01(0.58-1.75)$ & 0.97 & $0.98(0.61-1.57)$ & 0.93 \\
\hline \multicolumn{5}{|l|}{ Resistance pattern } \\
\hline Pansensitive & Referent & & Referent & \\
\hline INH or RIF resistance & $1.11(0.58-2.13)$ & 0.76 & $1.38(0.81-2.37)$ & 0.24 \\
\hline Multidrug resistance & $1.24(0.70-2.22)$ & 0.46 & $1.66(1.05-2.62)$ & 0.03 \\
\hline Other & $1.34(0.77-2.33)$ & 0.31 & $1.97(1.27-3.06)$ & 0.002 \\
\hline
\end{tabular}

${ }^{*}$ Results of multivariable regression analysis using classifier of tandem repeats method. aOR, adjusted odds ratio; INH, isoniazid; RIF, rifampin; TB, tuberculosis. 
more than the increased risk that would accrue from multiple exposures.

A review of the literature on factors associated with within-host diversity revealed substantial variability between settings. Studies from Botswana and Taiwan found a higher prevalence of polyclonal infection among patients with MDR-TB $(10,11)$; however, studies from Vietnam and KwaZulu-Natal (South Africa) did not find this association $(12,13)$. It is possible that this association may be modified in the presence of HIV coinfection or that the ability to identify such an association is easier in areas where the prevalence of multidrug resistance is higher.

The main strengths of this study relate to the large prospective cohort of pulmonary TB patients evaluated in a study area with a population of 3.3 million persons. However, $30 \%$ of enrolled participants did not have culture-confirmed TB, precluding MIRU-VNTR analysis on all participants. Use of the MIRU-VNTR assay on cultured specimens to detect within-host heterogeneity was motivated by practical considerations. Because MIRU-VNTR typing is unable to identify all minority variants, and some diversity may be lost during culture (14), our categorization of infections into simple, clonal, and polyclonal may be subject to misclassification, which would be differential (i.e., complex infections are more likely to be misclassified as simple than the reverse) and could lead to bias. The use of a high number of MIRUVNTR loci also reduces the likelihood of homoplasy. Furthermore, although the biological clock of the MIRU-VNTR marker seems to be relatively stable (recently estimated MIRU-VNTR mutation rate for TB is $2.70 \times 10^{-3}$ mutations/ locus/year [15]), changes accruing in the marker could lead to misclassification of clonal strains as polyclonal strains; we used the ClassTR method in an attempt to minimize such misclassification.

We found complex infections attributable to multiple infection events to be associated with increased risk for MDR TB. This finding further emphasizes the value of efforts to mitigate the transmission of MDR TB.

R.R.N. was supported by a Scholar Award from the Harvard Center for AIDS Research (National Institutes of Health [NIH] National Institute of Allergy and Infectious Diseases 2P30AI060354-11) and an Imperial College Global Health Institutional Strategic Support Fund fellowship from the Wellcome Trust. C.X.S. was supported by awards T32MH020031 and P30MH062294 from the NIH National Institute of Mental Health. L.C. was supported by a Natural Sciences and Engineering Research Council of Canada Discovery Grant and a Sloan Foundation Fellowship. L.C. and T.C. received support from an award from the NIH National Institute of General Medical Sciences (U54GM088558). L.C., R.C., Z.Z., J.G., C.C., R.Y., L.L., M.C.B., M.B.M., T.C., and collection of all data presented were supported by NIH (U01AI057786, U19A1076217).
Dr. Nathavitharana is an infectious diseases physician and research fellow at Beth Israel Deaconess Medical Center and Harvard Medical School, in Boston, Massachusetts, USA. Her primary research interests include using novel diagnostic strategies to characterize and decrease TB transmission.

\section{References}

1. Zetola NM, Shin SS, Tumedi KA, Moeti K, Ncube R, Nicol M, et al. Mixed Mycobacterium tuberculosis complex infections and false-negative results for rifampin resistance by GeneXpert MTB/ RIF are associated with poor clinical outcomes. J Clin Microbiol. 2014;52:2422-9. http://dx.doi.org/10.1128/JCM.02489-13

2. Zetola NM, Modongo C, Moonan PK, Ncube R, Matlhagela K, Sepako E, et al. Clinical outcomes among persons with pulmonary tuberculosis caused by Mycobacterium tuberculosis isolates with phenotypic heterogeneity in results of drug-susceptibility tests. J Infect Dis. 2014;209:1754-63. http://dx.doi.org/10.1093/infdis/ jiu040

3. Balmer O, Tanner M. Prevalence and implications of multiplestrain infections. Lancet Infect Dis. 2011;11:868-78. http://dx.doi.org/10.1016/S1473-3099(11)70241-9

4. Borgdorff MW, van Soolingen D. The re-emergence of tuberculosis: what have we learnt from molecular epidemiology? Clin Microbiol Infect. 2013;19:889-901. http://dx.doi.org/10.1111/ 1469-0691.12253

5. Cohen T, van Helden PD, Wilson D, Colijn C, McLaughlin MM, Abubakar I, et al. Mixed-strain Mycobacterium tuberculosis infections and the implications for tuberculosis treatment and control. Clin Microbiol Rev. 2012;25:708-19. http://dx.doi.org/ 10.1128/CMR.00021-12

6. World Health Organization. Global tuberculosis report 2016 [cited 2017 Apr 14]. http://www.who.int/tb/publications/global_report/en/ index.html

7. Zelner JL, Murray MB, Becerra MC, Galea J, Lecca L, Calderon R, et al. Bacillus Calmette-Guérin and isoniazid preventive therapy protect contacts of patients with tuberculosis. Am J Respir Crit Care Med. 2014;189:853-9. http://dx.doi.org/ 10.1164/rccm.201310-1896OC

8. Chindelevitch L, Colijn C, Moodley P, Wilson D, Cohen T. ClassTR: classifying within-host heterogeneity based on tandem repeats with application to Mycobacterium tuberculosis infections. PLOS Comput Biol. 2016;12:e1004475. http://dx.doi.org/10.1371/ journal.pcbi.1004475

9. Supply P, Allix C, Lesjean S, Cardoso-Oelemann M, Rüsch-Gerdes S, Willery E, et al. Proposal for standardization of optimized mycobacterial interspersed repetitive unit-variablenumber tandem repeat typing of Mycobacterium tuberculosis. J Clin Microbiol. 2006;44:4498-510. http://dx.doi.org/10.1128/ JCM.01392-06

10. Shin SSMC, Modongo C, Ncube R, Sepako E, Klausner JD, Zetola NM. Advanced immune suppression is associated with increased prevalence of mixed-strain Mycobacterium tuberculosis infections among persons at high risk for drug-resistant tuberculosis in Botswana. J Infect Dis. 2015;211:347-51. http://dx.doi.org/10.1093/infdis/jiu421

11. Huang HY, Tsai YS, Lee JJ, Chiang MC, Chen YH, Chiang CY, et al. Mixed infection with Beijing and non-Beijing strains and drug resistance pattern of Mycobacterium tuberculosis. J Clin Microbiol. 2010;48:4474-80. http://dx.doi.org/10.1128/ JCM.00930-10

12. Huyen MNKK, Kremer K, Lan NT, Cobelens FG, Buu TN, Dung NH, et al. Mixed tuberculosis infections in rural South Vietnam. J Clin Microbiol. 2012;50:1586-92. http://dx.doi.org/ 10.1128/JCM.00434-12 
13. Cohen T, Wilson D, Wallengren K, Samuel EY, Murray M. Mixedstrain Mycobacterium tuberculosis infections among patients dying in a hospital in KwaZulu-Natal, South Africa. J Clin Microbiol. 2011;49:385-8. http://dx.doi.org/10.1128/JCM.01378-10

14. Martín A, Herranz M, Ruiz Serrano MJ, Bouza E,

García de Viedma D. The clonal composition of Mycobacterium tuberculosis in clinical specimens could be modified by culture. Tuberculosis (Edinb). 2010;90:201-7. http://dx.doi.org/10.1016/ j.tube.2010.03.012
15. Ragheb MN, Ford CB, Chase MR, Lin PL, Flynn JL, Fortune SM. The mutation rate of mycobacterial repetitive unit loci in strains of M. tuberculosis from cynomolgus macaque infection. BMC Genomics. 2013;14:145. http://dx.doi.org/10.1186/1471-2164-14-145

Address for correspondence: Ruvandhi R. Nathavitharana, Beth Israel Deaconess Medical Center-Infectious Diseases, 110 Francis St, Ste GB, Boston, MA 02215-5501, USA; email: rnathavi@bidmc.harvard.edu

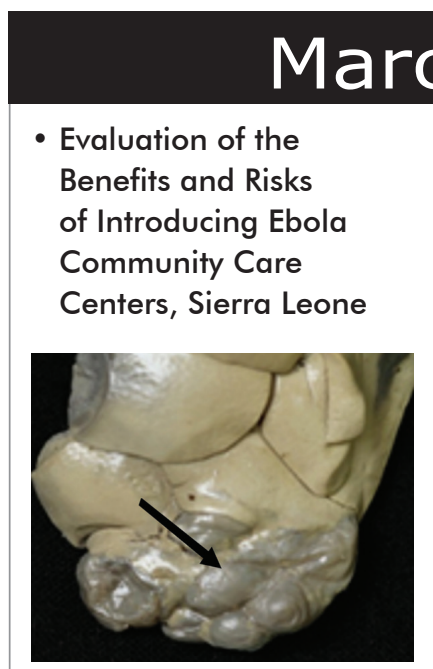

- Nanomicroarray and Multiplex Next Generation Sequencing for Simultaneous Identification and Characterization of Influenza Viruses

- Multidrug-Resistant Tuberculosis in Europe, 2010-2011

- Risk Factors for Death from Invasive Pneumococcal Disease, Europe, 2010

\section{- Mycoplasma} pneumoniae and Chlamydia spp. Infection in Community-Acquired Pneumonia, Germany, 2011-2012

- Epidemiology of Human Mycobacterium bovis Disease, California, USA, 2003-2011
- Regional Spread of Ebola Virus, West Africa, 2014

- Spillover of Mycobacterium bovis from Wildlife to Livestock, South Africa

- Prisons as Reservoir for Community Transmission of Tuberculosis, Brazil

- Polycystic Echinococcosis in Pacas, Amazon Region, Peru

- Spatiotemporal Analysis of Guaroa Virus Diversity, Evolution, and Spread in South America

- Red Deer as Maintenance Host for Bovine Tuberculosis, Alpine Region

- Noninvasive Test for Tuberculosis Detection among Primates

- Vertical Transmission of Bacterial Eye Infections, Angola, 2011-2012

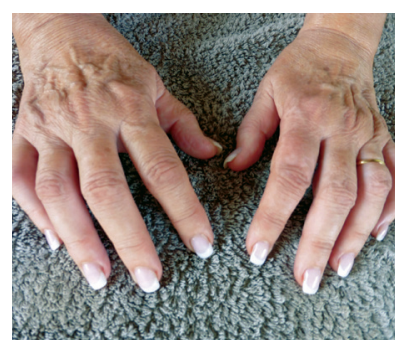

- Increased Risk for Multidrug-Resistant Tuberculosis in Migratory Workers, Armenia

- Endemic and Imported Measles VirusAssociated Outbreaks among Adults, Beiijing, China, 2013

- Mycobacterium bovis Infection in Humans and Cats in Same Household, Texas, USA, 2012

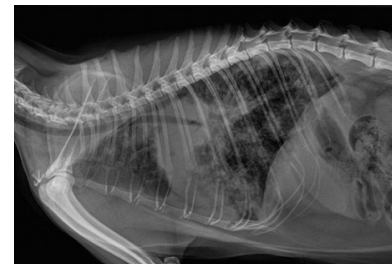

- Reemergence of Murine Typhus in Galveston, Texas, USA, 2013

- Severe Fever with Thrombocytopenia Syndrome in Japan and Public Heath Communication

- Novel Mutations in K13 Propeller Gene of Artemisinin-Resistant Plasmodium falciparum

- Comparison of Porcine Epidemic Diarrhea Viruses from Germany and the United States, 2014

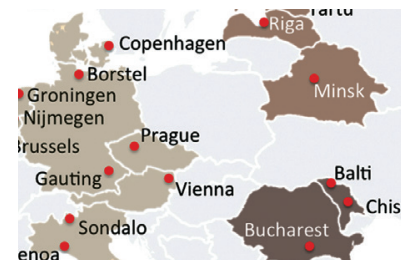

- Buruli Ulcer in Traveler from Suriname, South America, to the Netherlands

- Moxifloxacin Prophylaxis Against MDR TB, New York, New York, USA

- Rapid Detection of ESBL-Producing Enterobacteriaceae in Blood Cultures

- Characteristics of Tuberculosis Cases that Started Outbreaks in the United States, 2002-2011

- Reassortant Highly Pathogenic Influenza A(H5N6) Virus in Laos

- Autochthonous Dengue Fever, Tokyo, Japan, 2014

- Treatment of Ebola Virus Infection with Antibodies from Reconvalescent Donors

- Tuberculosis Microepidemics among Dispersed Migrants, Birmingham, UK, 2004-2013 